\title{
Detectors for High Count Rate Measurements with a Compensation for MPPC Gain Dependence on Temperature
}

\author{
A. Urban, A. Brosławski, G. Bołtruczyk, M. Gosk, S. Korolczuk, D. Rybka, V. Kiptily, M. Nocente, D. Rigamonti, \\ M. Tardocchi, I. Zychor and JET Contributors*
}

Multi Pixel Photon Counter (MPPC) is one of devices called silicon photomultiplier (SiPM). It is characterized by a fast response time, high gain coefficient, high photon detection efficiency resulting in good energy resolution, low voltage operation, resistance to mechanical shocks, compactness and immunity to a magnetic field. A MPPC gain is temperature dependent, so it is necessary to use a device which allows to maintain a constant value of a MPPC gain.

We report on two devices designed at the National Centre for Nuclear Research (NCBJ): FilterBox@NCBJ and MTCD@NCBJ to be used at the Joint European Torus (JET) during high count rate measurements.

The experiments at JET during D-T campaigns will be performed in harsh radiation conditions, especially at high rates up to $\sim 1 \mathrm{Mcps}$ and at relatively high gamma-ray energy about a few $\mathrm{MeV}$. Devices designed to replace existing detector at JET have to fit to available space and must use cabling installed almost 20 years ago. The device should be user-friendly and easy operated by both engineers and physicists. It is worth to notice that in case of a completely new system, some new designed elements could be optimized in comparison with a presented solution.

A device for real-time temperature monitoring and MPPC gain stabilization was designed and produced at NCBJ,

This scientific work was partly supported by Polish Ministry of Science and Higher Education within the framework of the scientific financial resources in the years 2015-2017 allocated for the realization of the international co-financed project. This work has been carried out within the framework of the EUROfusion Consortium and has received funding from the Euratom research and training programme 2014-2018 under grant agreement No 633053. The views and opinions expressed herein do not necessarily reflect those of the European Commission.

Arkadiusz Urban, Andrzej Broslawski, Grzegorz Boltruczyk, Marcin Gosk, Stefan Korolczuk, Dominik Rybka, and Izabella Zychor (e-mail: arkadiusz.urban@ncbj.gov.pl) are with Narodowe Centrum Badań Jądrowych (NCBJ), 05-400 Otwock, Poland.

Vasily Kiptily is with Culham Centre for Fusion Energy, Culham, United Kingdom.

Massimo Nocente and Davide Rigamonti are with Dipartimento di Fisica “G. Occhialini”, Università degli Studi di Milano-Bicocca,Milano, Italy.

Massimo Nocente, Davide Rigamonti and Marco Tardocchi are with Istituto di Fisica del Plasma "P. Caldirola", CNR, Milano, Italy.

*See the author list of "Overview of the JET results in support to ITER" by $\mathrm{X}$. Litaudon et al. to be published in Nuclear Fusion Special issue: overview and summary reports from the 26th Fusion Energy Conference (Kyoto, Japan, 17-22 October 2016). necessary due to a very strong voltage-temperature dependence characteristic for MPPC.

In Fig. 1, the overall scheme of electronics for the upgraded system is shown.

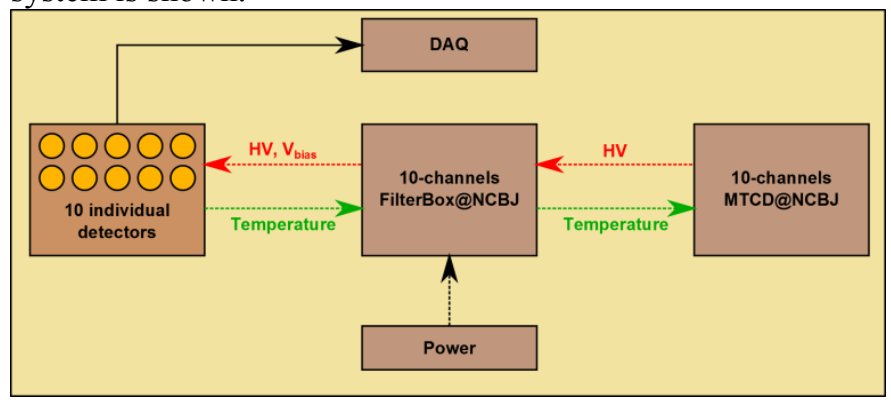

Fig. 1. Overall scheme of a detector system for the upgraded JET Gamma-ray Camera. HV denotes a bias voltage for MPPC, LV power supply for both temperature sensor and active elements.

The most important requirements for electronics could be summarized as follows:

- $\quad$ setting of MPPC bias voltage,

- integrated temperature sensor,

- $\quad$ integrated power supply MAX1932,

- $\quad$ advanced power supply closed loop control.

A detector system consists of a set of individual capsules with a scintillator and a dedicated printed circuit board (PCB). On each PCB, a temperature sensor is mounted. The TSIC $506 \mathrm{~F}$ sensor is characterized by an outstanding accuracy of $\pm 0.1 \mathrm{~K}$, an excellent long-term stability and a very low current consumption of $30 \mu \mathrm{A}$ during operation. The MPPCs, type S13361-3050NE-04 from Hamamatsu, were used.

An active system based on a transimpedance amplifier (TIA) was designed for MPPC signal read-out to obtain a signal characterized by a high output amplitude with low timeconstant. TIA is mounted on the same PCB described above.

In Fig. 2 a detailed schematic of TIA is shown. All results presented in this paper were obtained with the same experimental conditons and a CAEN Desktop Digitizer DT5730 was used for data acquisition. 


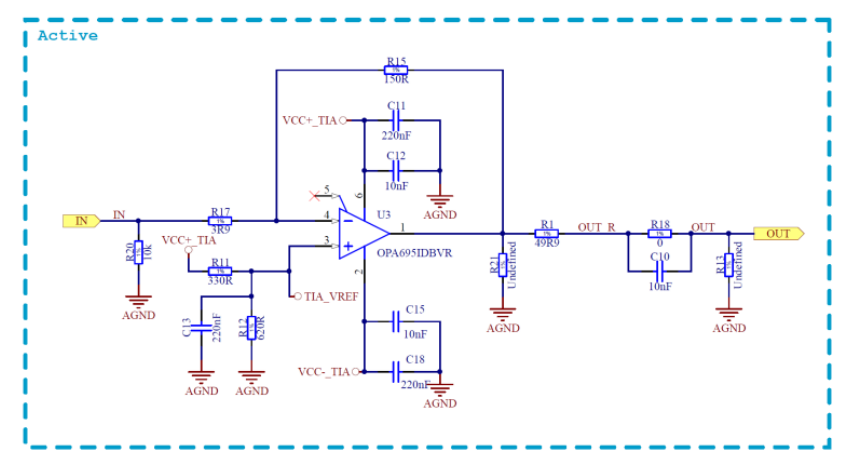

Fig. 2. Detailed schematic of TIA.

Information on temperature values is sent from a capsule to the FilterBox@NCBJ, based on a Microsemi ProAsic3 FPGA.

In Fig. 3 a photo of FilterBox@NCBJ is presented.

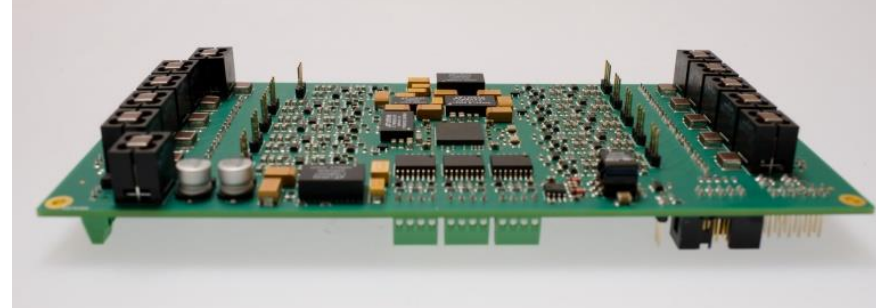

Fig.3 FilterBox@NCBJ.

The main functions of the FilterBox@NCBJ are:

1. to serve for all individual capsules in each part of the Gamma-ray camera,

2. to filter a bias voltage for MPPC in a capsule,

3. to power active elements, e.g., TIA and a temperature sensor, mounted on PCB. For each capsule, a separated DC linear voltage regulator is used to minimize noise and crosstalk between detectors,

4. to provide communication with the MTCD@NCBJ using three independent communication channels based on a RS485 standard. Via RS485 actual detector temperature values are read from FILTERBOX@NCBJ.

FilterBox@NCBJ is supplied from a dedicated low voltage laboratory power supply.

The firmware for the Microsemi ProAsic3 FPGA on FilterBox@NCBJ was designed in a VHDL language. It incorporates modules for reading a temperature from 10 sensors. The power supply for each temperature sensor can be switched on/off individually. Temperature readout is done continuously with a programmed frequency and temperature values are accessible by reading dedicated registers.

The MPPC Temperature Compensation Device (MTCD@NCBJ) is using a measured dependence of a breakdown voltage on temperature to maintain a constant value of the MPPC gain. The MPPC Temperature Compensation Device (MTCD@NCBJ) with integrated power supplies comprises two main parts: one is connected with 10 adjustable MPPC bias voltage channels for each individual capsule, the other one is used to determine an optimal value of a bias voltage which guarantees a constant gain. Each channel has its own isolated converter to eliminate ground loops, followed by a low-dropout regulator to minimize a ripple on output. Connections are protected against electrostatic discharges. All functions are controlled from a personal computer.

In Fig. 4 the MTCD@NCBJ board is shown.

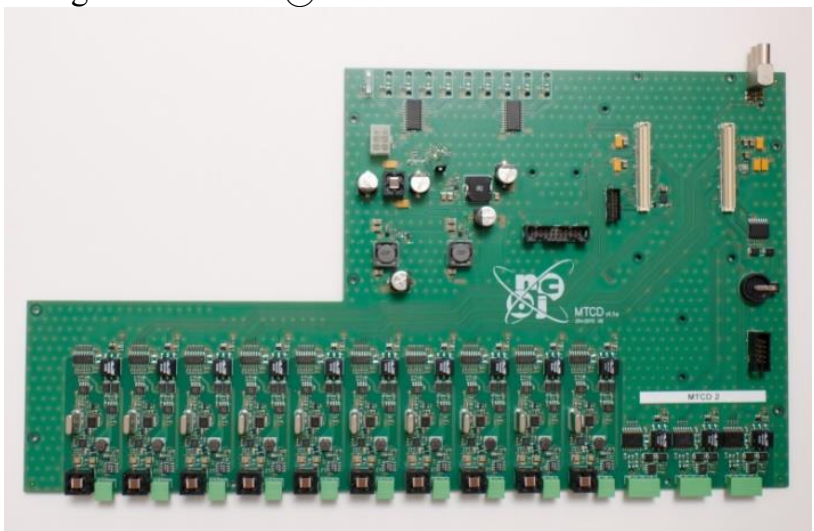

Fig. 4. MTCD@NCBJ with integrated power supplies.

Power supplies used in MTCD@NCBJ are characterized by an output voltage up to $80 \mathrm{~V}$, filtering of the MPPC voltage and an option to limit current.

A development board MicroZed based on the Xilinx Zynq ${ }^{\circledR}-7000$ was mounted in MTCD@NCBJ. A semiconductor device Zynq contains dual core ARM CortexA9 CPU with FPGA peripherals what allows to implement firmware both in HDL and high level language. A main algorithm was implemented in $\mathrm{C}$ language under Linux operating system to provide in an easy way a connection to MTCD@NCBJ via Ethernet or USB and investigation of an actual status of the device.

A control of 10 analog-to-digital (ADC) and 10 digital-toanalog (DAC) converters was implemented in VHDL. Modules allows to be controlled by CPU via standard AXI(AMBA) on chip interconnection.

PID (proportional-integral-derivative) regulator is applied to calculate a difference between actual and desirable voltage value. PID algorithm was implemented in $\mathrm{C}$ to control each high-voltage channel separately. A desirable value of a voltage is calculated based on temperature read from FilterBox@NCBJ.

In Fig. 5 a logic of MTCD@NCBJ is shown.

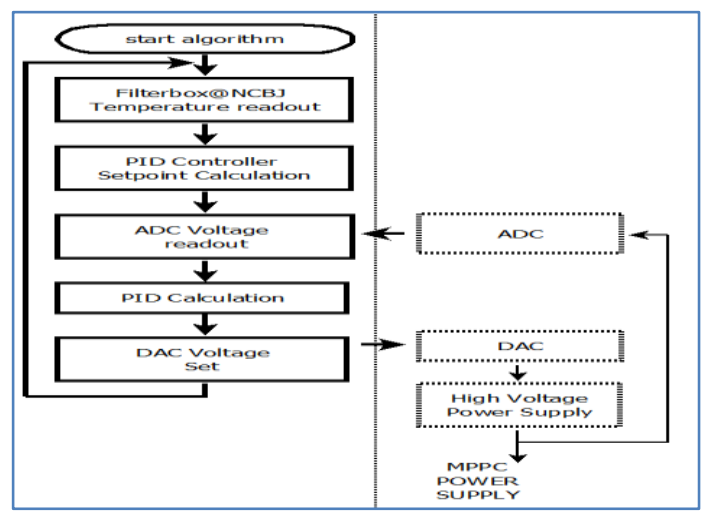

Fig. 5. Flow diagram of MTCD@NCBJ logic: left: CPU, right: programmable logic side in control program. 
A protection of overvoltage was implemented in a control part of a program to guarantee that detectors will not be destroyed by applying too high voltage.

The output voltage, determined by a 20-bits digital-analog converter (DAC1220E), is delivered through the buffer to the feedback loop of the HV switching regulator (MAX1932ETC+).

Measurements detailed:

- $20 \mathrm{~mm} \times 15 \mathrm{~mm}$ cylindrical $\mathrm{CeBr}_{3}$ scintillator,

- MPPC type S13361-3050NE-04 from Hamamatsu,

- active system based on a transimpedance amplifier (TIA) to obtain a signal characterized by a high output amplitude with low time-constant,

- $\quad{ }^{137} \mathrm{Cs}$ source emitting $661.7 \mathrm{keV}$ gamma linne with an activity of $400 \mathrm{MBq}$.

Preliminary results are presented in Figs. 6 and 7 as well as in Table 1.

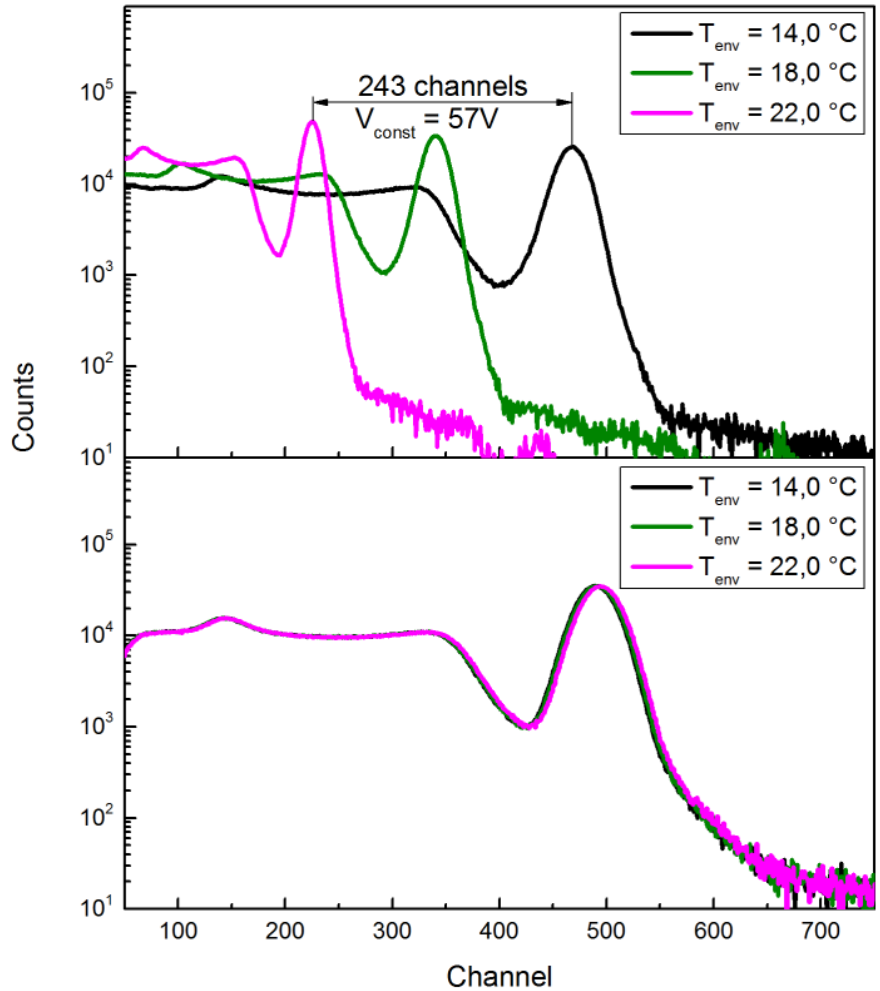

Fig. 6. Peak position as a function of MPPC temperature.

Upper: without MTCD@NCBJ.

Lower: with MTCD@NCBJ.

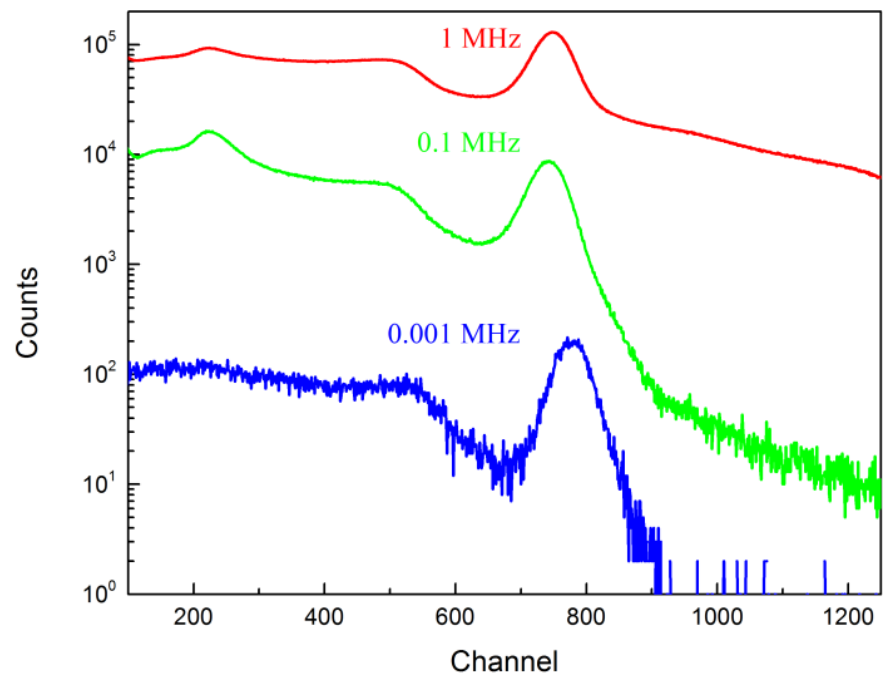

Fig. 7. Peak position as a function of count rate.

Table 1. Peak position and FWHM as a function of count rate.

\begin{tabular}{|l|l|l|l|}
\hline Rate, Mcps & $\begin{array}{l}\text { Peak } \\
\text { position } \\
(\mathrm{PP}), \mathrm{ch}\end{array}$ & $\begin{array}{l}\Delta=\mid \mathrm{PP}- \\
\mathrm{PP}_{\mathrm{av}} / \mathrm{PP}_{\mathrm{av}}, \%\end{array}$ & FWHM, \% \\
\hline 1 & 748 & 2.5 & 8.48 \\
\hline 0.1 & 743 & 3.3 & 8.46 \\
\hline 0.001 & 778 & 1.2 & 7.65 \\
\hline
\end{tabular}

\section{REFERENCES}

I. Zychor et al., "High performance detectors for upgraded gamma ray diagnostics for jet DT campaigns", Physica Scripta vol. 91, 064003, 2016

- G.Boltruczyk et al., "Development of MPPC+based detectors for high count rate DT campaigns at JET", Fusion Engineering and Design, 2017.

- A. Pellegrino, "Summary Actel ProAsic/3E Iradiation Tests", Actel Corporation, Nikhef, OT FE Architecture Mini-Review March 2013.

- S. Rezgui, "Radiation-Tolerant ProASIC3 FPGAs Radiation Effects", Actel Corporation, Tech. Report, April 2010.

- AVNET, "MicroZed Evaluation Kit", datasheet 2017.

- Hamamatsu Photonics K.K., "MPPC (Multi-Pixel Photon Counter) arrays," S13361-3050 series datasheet, April 2016.

- CAEN Electronic Instrumentation, Digitizer DT5730 datasheet [rev May 2016]. 